\title{
Modal analysis of the tugboat propeller in reduced scale
}

\author{
César A. Chagoyén-Méndez ${ }^{1}$, Haianny Pereira-Brito ${ }^{1}$, Jorge L. Moya-Rodríguez ${ }^{2}$, Ángel S. Machado- \\ Rodríguez $^{3}$; Yamill S. Campos-Pérez ${ }^{2}$
}

\author{
${ }^{1}$ Universidade Estadual do Amazonas (UEA), Escola Superior de Tecnologia (EST). Av. Darcy Vargas 1200.Parque Dez. Manaus-Amazonas-Brasil.CEP \\ :69050-020 \\ ${ }^{2}$ Instituto de Tecnologia e Educação Galileo da Amazônia (ITEGAM). Av. Joaquim Nabuco No 1950. Centro, Manaus - AM. CEP: 69005-080 \\ ${ }^{3}$ Universidad Central "Marta Abreu" de Las Villas (UCLV). Carretera a Camajuaní km 5.5, Santa Clara, Villa Clara, Cuba. CP 52830. \\ Email:cachagoyen@uclv.edu.cu, jorgemoyar@gmail.com, ycampos2525@gmail.com, asilviomr@gmail.com
}

Received: September $18^{\text {th }}, 2016$

Accepted: November $25^{\text {th }}, 2016$

Published: December $22^{\text {th }}, 2016$

Copyright (C2016 by authors and Institute of Technology Galileo of Amazon (ITEGAM) This work is licensed under the Creative Commons Attribution International License (CC BY 4.0).

http://creativecommons.org/licenses/by/4.0/ (c) (1) (2) Opea Acte:

\begin{abstract}
The ship under study belongs to the research prototyping Jaraqui, who won the award in the "Desafio Universitário de Nautidesign". Prior to the competition the ship modeling was performed in the DELFTship software to obtain the coefficients of the ship, following the rules of the competition. The diameter of the helix, the pitch, the speed, towing capacity or Bollard Pull (BP) for various percentages of operations were then determined. With these data propeller four models were built in the Solidworks software with diameters of 9 and $10.8 \mathrm{~cm}, 3$ and 4 blades. For the correct location of the loads the principles of naval architecture regarding the propulsive thrust loads and according to the radius of the propeller (Diagram Burrill) were taken into account. The modal analysis was performed on Inventor software give modes and natural oscillation frequencies so as to operating loads. From these analyzes it is concluded that the resonance phenomenon does not occur in any of the propellers analyzed.
\end{abstract}

Keywords: Propeller; Modal analysis; Tugboat g.

Análisis modal de la hélice de un remolcador en escala reducida

\begin{abstract}
RESUMEN
La embarcación objeto de estudio pertenece a la línea de investigación de construcción de prototipos Jaraqui, que obtuvo premio en el "Desafio Universitário de Nautidesign". Previo a la competencia se realizó el modelado de la embarcación en el software DelftShip para obtener los coeficientes de la embarcación, cumpliendo las reglas de la competición. Se determinaron entonces el diámetro de la hélice, el paso, la velocidad de avance, la capacidad de remolque o Bollard Pull (BP) para distintos porcientos de operación. Con estos datos se construyeron cuatro modelos de hélices en el software Solidworks con diámetros de 9 y $10.8 \mathrm{~cm}$, de 3 y 4 palas. Para la correcta ubicación de las cargas se tuvieron en cuenta los principios de la arquitectura naval referidas a las cargas propulsivas y el empuje de acuerdo al radio del propulsor (Diagrama de Burril). El análisis modal se realizó en el software Inventor obteniéndose los modos y las frecuencias de oscilación tanto natural como ante las cargas de operación. De estos análisis se concluye que el fenómeno de resonancia no se presenta en ninguna de las hélices analizadas.
\end{abstract}

Palabras claves: Hélice; Análisis modal; Remolcador.

\section{INTRODUCCIÓN}

En Brasil existe una competición universitaria inédita denominada "DUNA Desafio Universitário de Nautimodelismo" creado por la Universidade Federal de Santa Catarina en el que participan universidades brasileñas y de otros países. Los universitarios son estimulados a desarrollar y construir un modelo funcional en escala reducida de una embarcación. Tiene como objetivo fomentar la generación de innovaciones en el área de Ingeniería Naval, así como la interacción entre los alumnos de esta carrera. Los modelos concebidos por los equipos son sometidos a una serie de pruebas durante varios días. $\mathrm{La} 3^{\circ}$ edición de esta competencia tuvo lugar del 24 al 27 de septiembre de 2015 en la ciudad de Joinville - SC, en el Recanto Ararat. Participaron 22 equipos y más de 200 universitarios. 
El equipo Jaraqui2 de la Universidade Estadual do Amazonas (UEA) dbtloøestlopeimete importancia fundamental minimizar el nivel de lugar con 396 puntos [1].

Este trabajo se centra en el análisis modal de la hélice de la embarcación que participó en esta competencia, de la cual se muestra una imagen a continuación.



Figura 1: Embarcación Jaraqui2.

Fuente: Equipo Jaraquí2, (2015).

Los modelos entendidos como representaciones en escala reducida han sido empleados en todas las épocas. Se admite que en sus orígenes los modelos de barcos tuvieron carácter ritual, siendo numerosos los ejemplares hallados. Quizás los más representativos son la embarcación de plata de Ur (3.000 años A.C.) aparecida en Caldea y las encontradas en la tumba de Tutankhamon. Gracias a estas y otras muestras, así como a los relieves, se tiene una idea cabal de los barcos de la antigüedad $[2,3]$

Los modelos navales, hasta épocas todavía recientes, han sido utilizados en la construcción de barcos. En la actualidad se distinguen básicamente dos clases o especialidades, el llamado estático, formado por barcos destinados a ser expuestos y el dinámico dotado de velas o motores y controles a distancia que se emplean sobre todo para juegos y competiciones, incluso internacionales en las que participan numerosos aficionados y que están regidas por una prolija reglamentación. Esta segunda especialidad se emplea también para la experimentación e investigación lo cual contribuye a disminuir los costos y los riesgos, lo que se corresponde con este trabajo. [4]

En el diseño de estructuras navales, uno de los aspectos que deben ser garantizados es un ambiente agradable para el trabajo continuo, eficiente y seguro de los equipos mecánicos, eléctricos y de los tripulantes. Un problema encontrado en las embarcaciones es la vibración, siendo la hélice una de las fuentes más comunes de excitación vibracional.

Vibración es el movimiento oscilatorio en torno a un punto de referencia. Independiente de su nivel y modo es indeseable, pues origina problemas como la desalineación de la línea de eje, comprometiendo la estructura del sistema de propulsión que ocasiona fallas en las subestructuras de la embarcación, en los componentes mecánicos, electrónicos, incomodidad a los usuarios y el movimiento de las cargas. Por vibración.

El estudio de las vibraciones en las embarcaciones es un tema de alta complejidad ya que las mismas pueden ser inducidas por diferentes fuentes como por ejemplo máquinas, equipamientos, olas, vientos y efectos hidrodinámicos.

Por otro lado, las herramientas computacionales han ganado espacio entre los ingenieros con el uso de modelos numéricos. Por tanto, el estudio de la vibración aplicada a la hélice, realizando su modelado y simulación es hoy una práctica que conlleva al desarrollo de embarcaciones con mayor confort, seguridad y menor costo.

En este trabajo se realiza el estudio del comportamiento vibratorio de la hélice propulsora convencional de una embarcación en escala reducida, verificando la aparición del fenómeno de resonancia para cuatro modelos de hélices con el objetivo de minimizar el efecto de la vibración.

\section{MATERIALES Y MÉTODOS}

Se realizó el modelado de la embarcación en escala reducida del tipo remolcador en el software DelftShip [5] del cual se obtuvieron los coeficientes de la embarcación tales como longitud total, calado, volumen desplazado, desplazamiento, longitud de la línea de agua, resistencia, etc. Posteriormente se realizó el modelo de la embarcación en AutoCAD [6] como se puede apreciar en la figura 1.

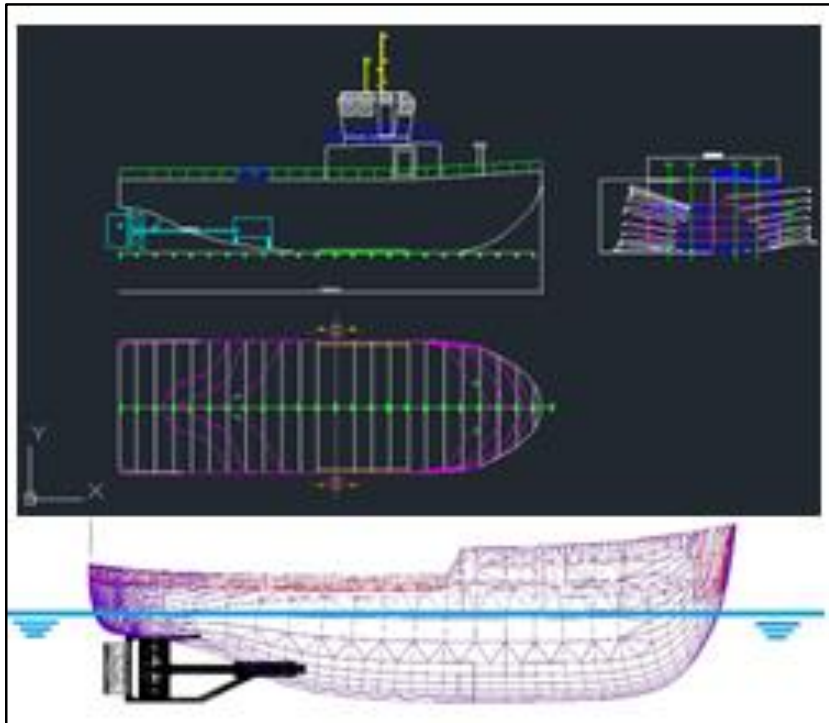

Figura 2: Modelo de la embarcación realizada en AutoCAD.

Fuente: Los autores, (2015).

Posteriormente se seleccionó el motor en base a las reglas de la competencia y se calculó el diámetro (D) de la hélice, el paso (P), la capacidad de remolque o Bollard Pull para distintos porcientos de operación y la velocidad de avance (Va). Se empleó el software Hélice [7] el cual permitió decidir qué hélices se simularían variando el diámetro y el número de palas. En la figura 2 aparece una imagen del modelado de una de las hélices. 


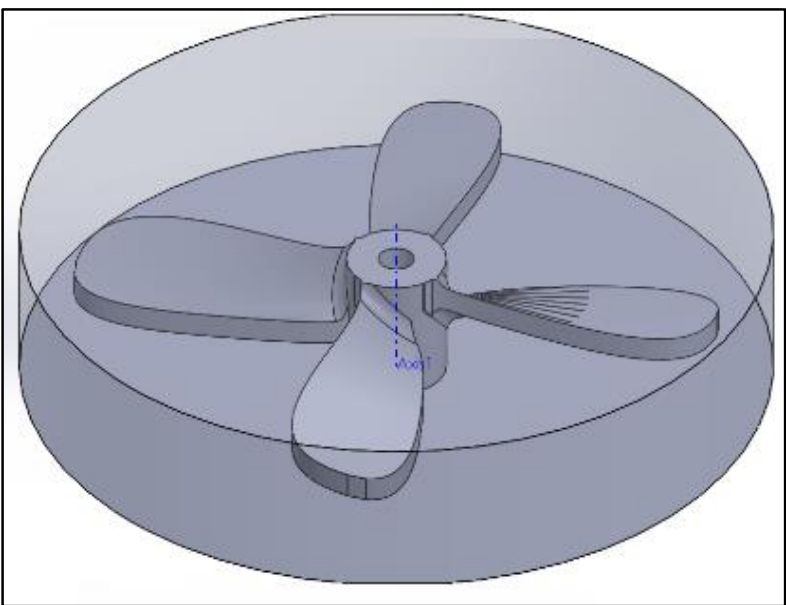

Figura 3: Modelado de una de las hélices.

Fuente: Los autores, (2015).

La geometría y dimensiones de las cuatro hélices a simular fue realizada en el software SolidWorks [8] las cuales son:

- Hélice I ( $9,0 \mathrm{~cm}-3$ palas)

- Hélice II $(9,0 \mathrm{~cm}-4$ palas $)$

- Hélice III (10,8 cm - 3 palas)

- Hélice IV (10,8 cm - 4 palas)

Su diseño se puede apreciar en las figuras 3-6.

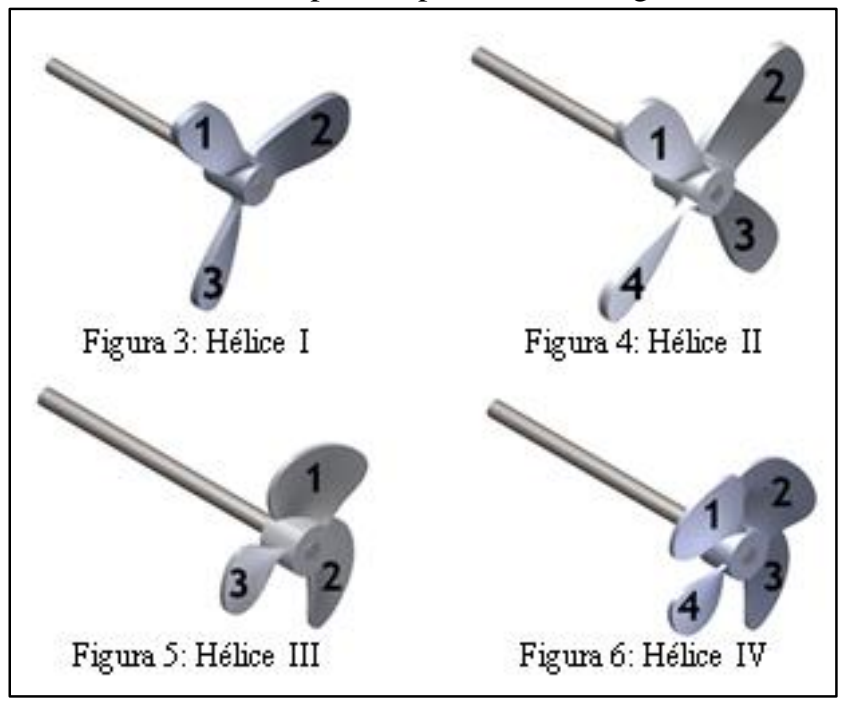

Figura 4: Modelos de Hélices tipo I, II, III e IV.

Fuente: Los autores, (2015).

El sistema de propulsión de la embarcación es compuesto esencialmente por el conjunto motor-árbol-hélice. En este trabajo se simuló parte del árbol y la hélice, utilizándose para ello el software Inventor [9]. Según los principios de la arquitectura naval el campo de presión de la hélice en el fluido no siempre es el mismo en todo el radio, siendo común que el campo en la punta sea reducido, influenciado por el efecto de la cavitación [10-12].

Las hélices al trabajar producen dos fuerzas que son: la fuerza de empuje y la fuerza propulsiva o lateral. A estas fuerzas se adiciona el torque para completar las cargas que actúan sobre el modelo elaborado de la hélice y el árbol [13-16].

La fuerza de empuje es paralela al eje y es la que impulsa la embarcación hacia delante, es decir, la hélice empuja el agua hacia atrás (acción) y a su vez es "empujada" hacia delante por una fuerza igual (reacción). La fuerza propulsiva o lateral es perpendicular al eje y tira de la popa hacia la misma banda a la que gira la hélice. La fuerza propulsiva se puede determinar a partir de la expresión siguiente:

$$
F=\frac{P}{V}
$$

donde:

$F$ - Fuerza propulsiva, en $N$.

$P$ - es la potencia propulsiva, en watts.

$V$ - es la velocidad crucero, en $\mathrm{m} / \mathrm{s}$.

Para esta embarcación, después de realizar un conjunto de cálculos y considerando el $80 \%$ de operación efectiva del motor, se determinó que la potencia a utilizar es de 744 watts y la velocidad crucero de $0.51 \mathrm{~m} / \mathrm{s}$. Como resultado se obtiene una fuerza propulsiva de $1458.8 \mathrm{~N}$.

De ese conjunto de cálculos y consideraciones se obtuvo que la fuerza de empuje alcanza un valor de 12,83 N.

El torque se determinó a partir de la expresión:

$$
T=\frac{P}{2 . \pi \cdot f}
$$

donde:

$T$ - es el Torque, en N.m.

$f$-son las rotaciones del motor, en rpm.

Después de realizado el cálculo se obtiene un torque de 0.12 N.m.

La fuerza propulsiva o lateral aparece localizada en el extremo de la pala. La fuerza de empuje se distribuye por todo el radio del propulsor. El torque se ubica en el árbol como se puede apreciar en la figura 5. En la figura 8 se puede apreciar la malla de elementos finitos de una de las hélices simuladas.

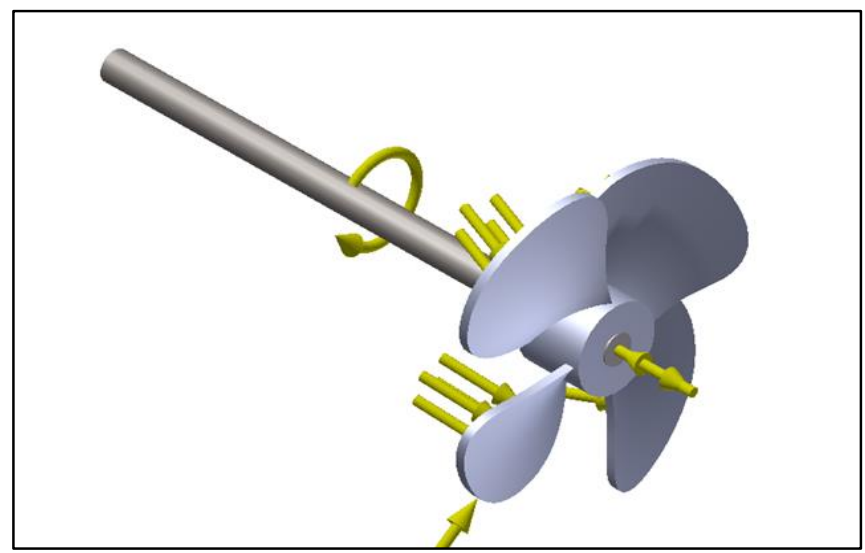

Figura 5: Modelo de hélice con las cargas aplicadas.

Fuente: Los autores, (2015). 
Silva et al., ITEGAM-JETIA. Vol.02, № 08, pp.114-120. Dezembro, 2016.

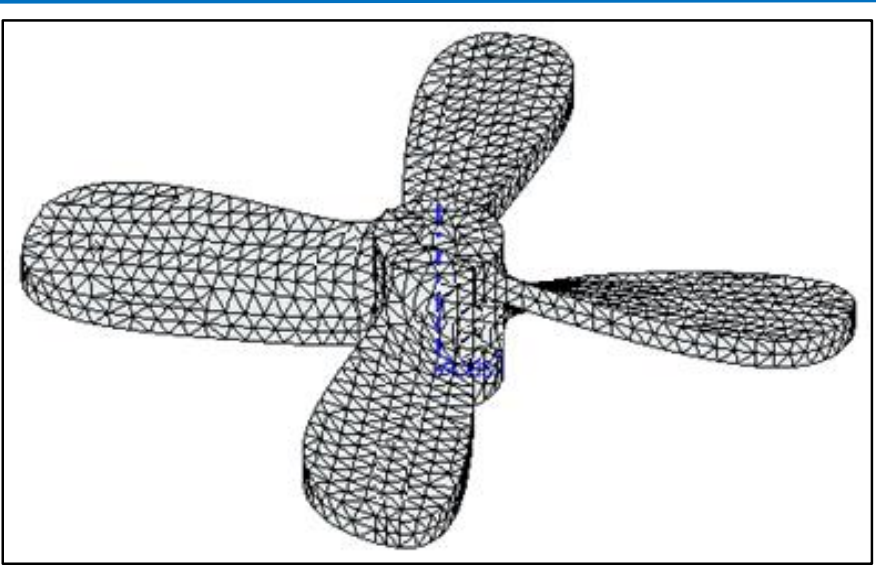

Figura 6: Malla de elementos finitos.

Fuente: Los autores, (2015).

El material utilizado para la fabricación de la hélice fue Aluminio 6061-T8 [17] mientras que el árbol es de acero al carbono AISI 1045 [18]. En la Tabla 1 aparecen algunas de sus propiedades Físico-Mecánicas.

Tabla 1: Algunas propiedades Físico-Mecánicas del Aluminio 6061-T8 y el AISI 1045.

\begin{tabular}{|l|c|c|}
\hline \multicolumn{1}{|c|}{ Propiedad } & $\begin{array}{c}\text { Aluminio } \\
\text { 6061-T8 }\end{array}$ & $\begin{array}{c}\text { AISI } \\
\mathbf{1 0 4 5}\end{array}$ \\
\hline Límite de Fluencia (MPa) & 276.0 & 690 \\
\hline Resistencia a la Tracción (MPa) & 310.0 & 620 \\
\hline Módulo de Young (GPa) & 68,9 & 206 \\
\hline Coeficiente de Poisson & 0,33 & 0.29 \\
\hline $\begin{array}{l}\text { Módulo de Cizallamiento } \\
\text { (MPa) }\end{array}$ & 26.0 & 80000 \\
\hline Densidad $\left(\mathrm{g} / \mathrm{cm}^{3}\right)$ & 2,7 & 7.85 \\
\hline
\end{tabular}

Para llevar a cabo el análisis fueron aplicadas a los cuatro modelos estudiados restricciones de montaje entre el árbol y la hélice, restricciones de movimiento tipo rotación, especificando el giro de la hélice y restricción del tipo pasador, fijándose las direcciones radial y axial del árbol y dejando libre la dirección tangencial.

\section{RESULTADOS Y DISCUSIÓN}

Para conocer el comportamiento modal de cada modelo de hélice, es necesario realizar el análisis bajo dos condiciones: free-free y con cargas y restricciones. Estos dos análisis permitirán conocer los modos y las frecuencias naturales de oscilación (freefree) y conocer el comportamiento modal más cercano a la realidad, es decir, bajo cargas y restricciones. [19-21].

Se verificó la aparición o no del fenómeno de resonancia. Este fenómeno aparece cuando la frecuencia de oscilación bajo cargas y restricciones se iguala a la frecuencia natural de oscilación. Por ello se calculó la diferencia entre ambos resultados, si es diferente de cero no aparece este perjudicial fenómeno.

Un modelo real tiene un número infinito de frecuencias naturales. Sin embargo, un modelo de elementos finitos tiene un número finito de frecuencias naturales que es igual al número de grados de libertad considerados en el modelo. Para la mayoría de los propósitos del cálculo de frecuencias sólo son necesarios los primeros modos.

Se determinaron las primeras cinco frecuencias y los correspondientes modos de oscilación o vibración de cada uno de los cuatro modelos de hélice.

En la tabla 2 se pueden apreciar los resultados numéricos del análisis, es decir, la frecuencia natural de oscilación y la frecuencia obtenida bajo cargas y apoyos, así como la diferencia entre ambas.

Tabla 2: Resultados numéricos del análisis de frecuencia.

\begin{tabular}{|l|l|l|l|}
\hline \multirow{2}{*}{$\begin{array}{l}\text { Nodo } \\
\text { No. }\end{array}$} & \begin{tabular}{l} 
Frecuencias Hélice I (Hz) \\
\cline { 2 - 3 } \\
Free-
\end{tabular} & $\begin{array}{l}\text { Con cargas y } \\
\text { restricciones }\end{array}$ & $\begin{array}{l}\text { Diferencia } \\
(\mathrm{Hz})\end{array}$ \\
\hline 1 & 619,62 & 756,10 & 136,9 \\
\hline 2 & 645,83 & 756,48 & 110,65 \\
\hline 3 & 1234,02 & 1010,24 & 223,78 \\
\hline 4 & 1236,90 & 2019,57 & 782,67 \\
\hline 5 & 1246,34 & 2019,70 & 773,36 \\
\hline Frecuencias Hélice II (Hz) \\
\hline 1 & 576,73 & 755,18 & 178,45 \\
\hline 2 & 593,57 & 761,31 & 167,74 \\
\hline 3 & 654,49 & 788,61 & 134,12 \\
\hline 4 & 1247,17 & 967,83 & 279,34 \\
\hline 5 & 1248,34 & 2010,97 & 762,63 \\
\hline Frecuencias Hélice III (Hz) \\
\hline 1 & 866,10 & 1296,44 & 430,34 \\
\hline 2 & 940,54 & 1374,96 & 434,42 \\
\hline 3 & 1454,26 & 1611,88 & 157,62 \\
\hline 4 & 1497,04 & 3369,35 & 1872,31 \\
\hline 5 & 1699,61 & 3390,62 & 1691,01 \\
\hline Frecuencias Hélice IV (Hz) \\
\hline 1 & 817,05 & 1306,53 & 489,48 \\
\hline 2 & 887,10 & 1323,65 & 436,55 \\
\hline 3 & 1309,64 & 1336,90 & 27,26 \\
\hline 4 & 1460,82 & 1499,92 & 39,1 \\
\hline 5 & 1474,15 & 3381,49 & 1907,34 \\
\hline
\end{tabular}

Fuente: Los autores, (2015).

De las cuatro hélices simuladas, la hélice IV es la que más cercana está a la ocurrencia del fenómeno de resonancia, particularmente en las frecuencias de los modos 3 y 4 en que la diferencia entre la frecuencia natural (free-free) y la frecuencia con cargas y restricciones es de 27.26 y $39.1 \mathrm{~Hz}$, respectivamente. En el resto de las hélices la diferencia es mayor de $100 \mathrm{~Hz}$. De forma general se puede decir que este fenómeno no aparece en ninguno de los cuatro modelos simulados.

En la figura 9 se muestran imágenes de los modos de oscilación natural y bajo cargas y restricciones de los cuatro modelos de hélice simulados.

Una descripción de los cuatro primeros modos de oscilación de los modelos de hélices analizados (figura 9), específicamente de los modos bajo cargas y con restricciones, es la siguiente: 


\section{Hélice I:}

Modo 1: ocurren oscilaciones de flexión mayormente de la pala 1 alrededor del eje $\mathrm{x}$.

Modo 2: aparece flexión alrededor del eje x predominantemente de la pala 3.

Modo 3: ocurren oscilaciones de flexión de todas las palas, un poco mayor de la pala 2 .

Modo 4: las palas 1 y 2 se flectan más que las otras dos palas, pero lo hacen en direcciones contrarias, es decir, una hacia delante y otra hacia atrás.

\section{Hélice II:}

Modo 1: ocurren oscilaciones de flexión mayormente de las palas 2 y 4 alrededor del eje $\mathrm{x}$ en direcciones contrarias, es decir, una hacia delante y la otra hacia atrás. La pala 3 también oscila, pero en menor medida.
Modo 2: las palas 1 y 3 oscilan en flexión más que las otras dos palas, también en direcciones contrarias.

Modo 3: las oscilaciones de la pala 4 son mayores que el resto de las palas, aunque éstas también oscilan.

Modo 4: la pala 1 es la que tiene mayor oscilación en flexión, el resto de las palas lo hacen, pero en menor medida.

\section{Hélice III:}

Modo 1: las oscilaciones de flexión ocurren mayoritariamente en la pala 1 y en dirección contraria a como lo hacen las palas 2 y 3 .

Modo 2: la pala 2 es la que más oscila, aunque la pala 3 también lo hace, pero en menor medida.

Modo 3: las oscilaciones de la pala 3 son mayores, aunque las palas 1 y 2 también oscilan en flexión.

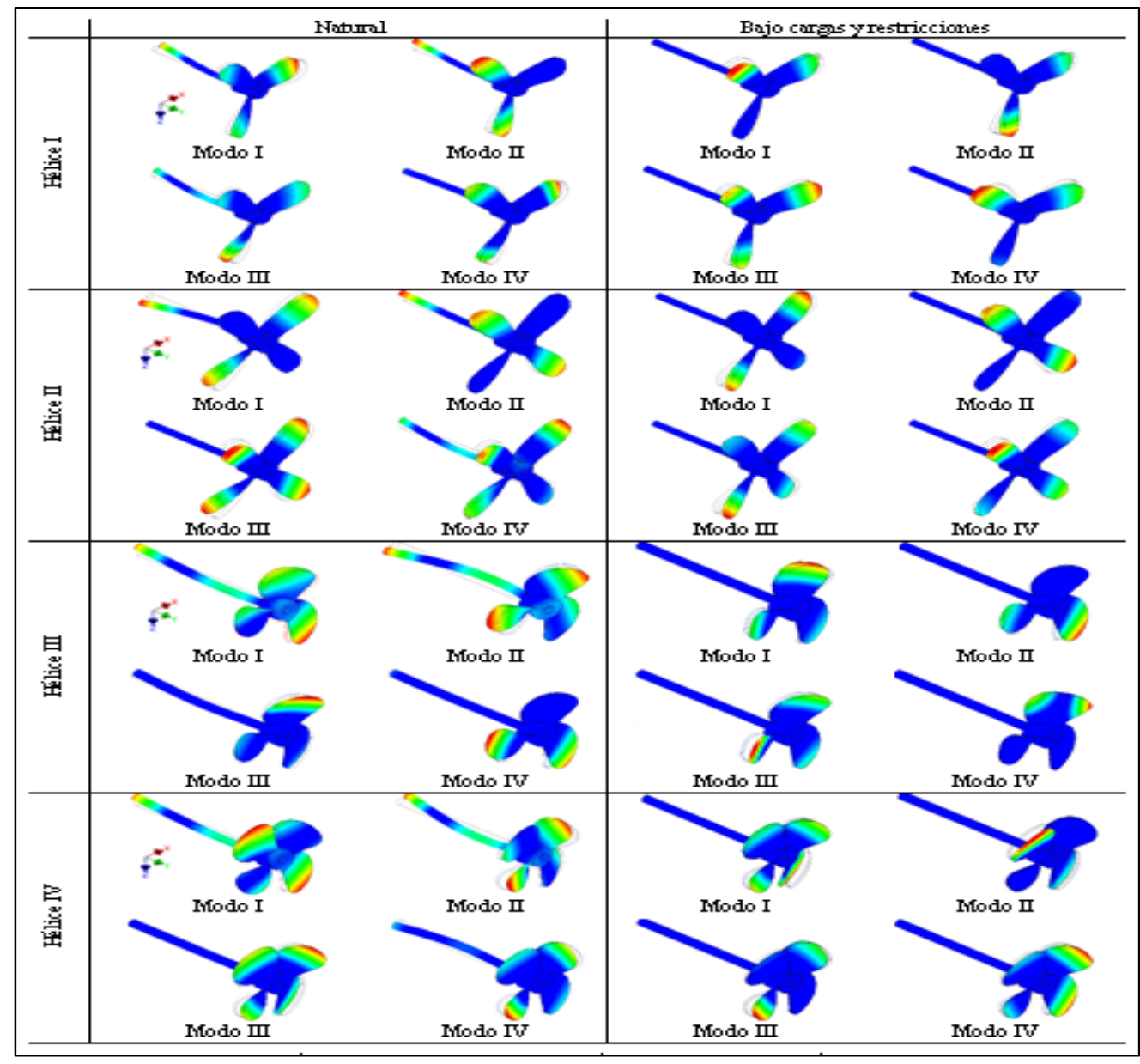

Figura 7: Imágenes de los modos de oscilación de los modelos de hélice simulados

Fuente: Los autores, (2015).

\section{Hélice IV}

Modo 1: todas las palas oscilan en flexión, pero la pala 3 lo hace más que el resto.

Modo 2: las palas 1 y 3 oscilan en flexión en direcciones contrarias, pero la 1 oscila más que la 3 .

Modo 3: las palas 2 y 4 oscilan en flexión en direcciones contrarias, pero la 4 oscila más que la 2 .
Modo 4: las cuatro palas oscilan en la misma dirección, pero mayoritariamente lo hacen la 2 y la 3.

Se puede resumir que la mayoría de las palas oscilan en flexión. De los cuatro primeros modos en solo uno, de un tipo de hélice aparece la torsión en una de las palas. 
Después de analizar detalladamente todos estos resultados se elige la hélice II para la embarcación, de la cual se hace una impresión $3 \mathrm{D}$ y posteriormente se funde en arena, como aparece en las figuras 8 y 9.

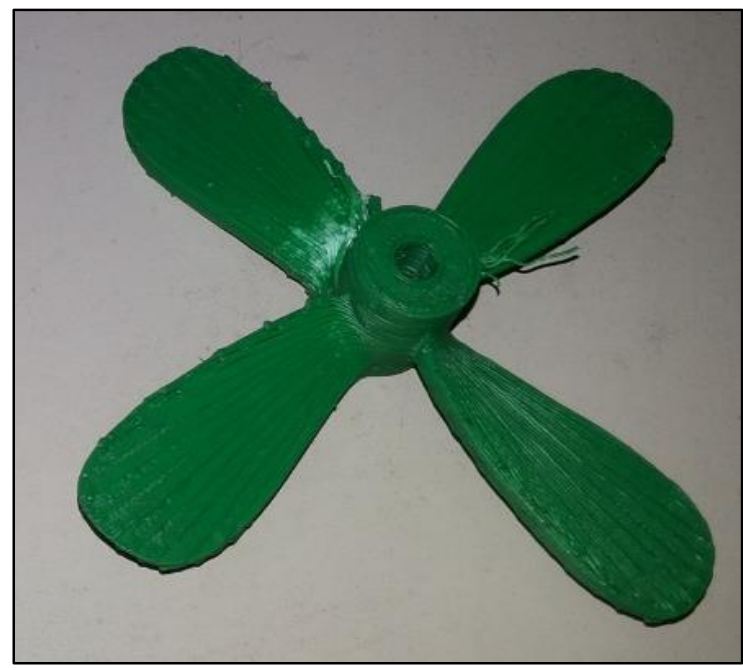

Figura 8. Impresión 3D de la Hélice II. Fuente: Los autores, (2015).

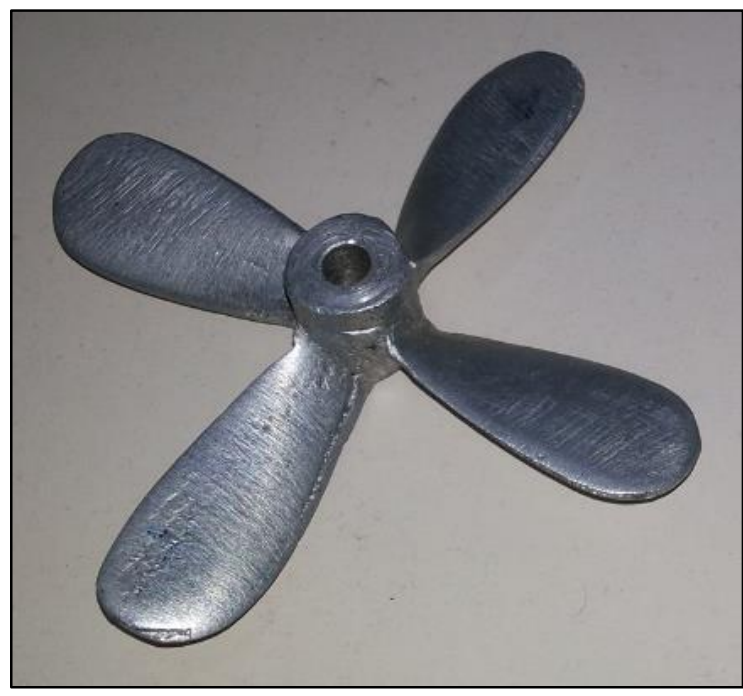

Figura 9. Modelo fundido en arena. Fuente: Los autores, (2015).

\section{CONCLUSIONES}

Del análisis modal de las hélices se obtuvieron las primeras frecuencias y modos de vibración tanto naturales como bajo cargas y restricciones, comprobándose la no ocurrencia del fenómeno de resonancia.

Esta metodología de cálculo y análisis puede aplicarse a embarcaciones de mayor envergadura.

A partir de la simulación animada de las hélices se pudo comprobar que la mayoría oscilan en flexión y en una sola aparece la torsión.
En base a todos estos resultados se eligió y fabricó la hélice II la cual fue utilizada en la embarcación durante la competencia.

\section{AGRADECIMIENTOS}

A Escuela Supperior del Tecnología de la Universidade do Estado do Amazonas (EST- UEA) y la Fundación del Amparo de la Pesquisa do Estadio del Amazonas (FAPEAM).

\section{REFERENCIAS BIBLIOGRÁFICAS}

[1] DUNA. Desafio Universitário de Nautimodelismo. Disponible en: http://www.oficialduna.com/duna2015/ Acceso en: Agosto 2015.

[2] Oficios Tradicionales. Dpto. para la Innovación y la Sociedad del Conocimiento 2011. Disponible en: http://www.oficiostradicionales.net/es/mar/navales/ Acceso en: Agosto 2015.

[3] Aguirre Sorondo, Antxon. Artisautza. Euskonews \& media. 2004/05/07-14.

[4] Chapa Ozamiz, Alfonso. Primer catálogo del modelismo naval en Vizcaya. La Gran Enciclopedia Vasca. Volumen VIII. Bilbao 1974.

[5] DelftShip. Marine Software. Netherlands, Disponible en: http://www.delftship.net/ Acceso en: Octubre 2015.

[6] AutoCAD 2016 Autodesk Inc. Disponible en: http://www.autodesk.com.br/ Acceso en: Octubre 2015.

[7] Programa Hélice. Sistema Propulsivo. Gabriel Taffarel y André Ramiro, Universidade Federal de Rio de Janeiro. Disponible en:

http://www.oceanica.ufrj.br/deno/prod_academic/relatorios/atuais/ Robledo-Giano/relat1/Sistema\%20Propulsivo.htm Acceso en: Octubre 2015.

[8] SolidWorks 2016. Dassault Systèmes. Disponible en: http://www.solidworksbrasil.com.br/ Acceso en: Noviembre 2015.

[9] Inventor 2016 Autodesk Inc. Disponible en: http://www.autodesk.com/products/inventor/ Acceso en:

Noviembre 2015.

[10] DEnO. Departamento de Engenharia Naval e Oceânica. Disponible en:

http://www.oceanica.ufrj.br/deno/prod_academic/relatorios/atuais/ Robledo-Giano/relat1/Sistema\%20Propulsivo.htm Acceso en: Octubre 2015. 
[11] Padovezi, C.M. Aplicação de Resultados de Escala Real no

Projeto de Hélices de Embarcações Fluviais. São Paulo, 1997.

[12] Cândido, I. S. Projeto Integrado de Sistema Propulsivo com

Requisito de Tração Estática. Rio de Janeiro, 2012.

[13] Holtrop, J. A Statistical Re-Analysis on Resistance and Propulsion Data - International Shipbuilding Progress, pp- 272276, 1984.

[14] Trindade, J. Hidrodinâmica e Propulsão. Enidh, 2012.

[15] Fonseca, Maurílio Magalhães, Arte Naval. - $6^{a}$ Ed. Rio de Janeiro: Serviço de Documentação Geral da Marinha, 2002. p. 902.

[16] Dokkun, K.V. The Maritime Engineering Reference Book: A Guide to Ship Design, Constrution and Operation. $5^{\text {a }} \mathrm{Ed}$. Burlington: Butterworth-Heinemann, 2008.

[17] Alumnio 6061-T8 MatWeb Copyright 1996-2016 by MatWeb, LLC. Disponible en:

http://matweb.com/search/DataSheet.aspx?MatGUID=90404a0c00 1c4016b2b359a6c19f9127, Aceso en: Noviembre 2015.

[18] AISI 1045 MatWeb Copyright 1996-2016 by MatWeb, LLC. Disponible en:

http://matweb.com/search/DataSheet.aspx?MatGUID=2551e9ac36 274110a7ab53be5ca585de, Acceso en: Noviembre 2015.

[19] Moliner, Lucas. Estudo do comportamento vibratório de uma embarcação em escala reduzida. 2014. 60p. Dissertação (Bacharelado em Engenharia Naval) - Universidade Federal de Santa Catarina, Santa Catarina, 2014. [Orientador: Thiago Antonio Fiorentin].

[20] Vale, A. R. M. Análise modal numérico-experimental de hélices navais produzidos na região Amazônica. 2003. 169f. Trabalho de Conclusão de Curso (Bacharelado em Engenharia Mecânica) - Universidade Federal do Pará, Pará. [Orientador: Prof. Dr. Newton Sure Seiro].

[21] Lamb, T. Ship Design and Construction I e II, New Jersey, USA: The Society of Naval Architects and Marine Engineers One World Trade Center. p. 733. 